\title{
CHARACTERIZATION OF THE PROTON BEAM FROM THE 6.7 MEV LEDA RFQ*
}

\author{
M.E.Schulze ${ }^{\dagger}$, General Atomics, Los Alamos, NM, 87544, USA \\ C.K.Allen, P.L. Colestock, J.D.Gilpatrick, W. Lysenko, L.J. Rybarcyk, J.D.Schneider, \\ R.L.Sheffield, H.V.Smith, and T.P.Wangler \\ LANL, Los Alamos, NM 87545, USA \\ K.R.Crandall, TechSource, Santa Fe, NM 87594-1057 USA
}

\begin{abstract}
The present configuration of the Low-Energy Demonstration Accelerator (LEDA) consists of a 75-keV proton injector, a 6.7-MeV 350-MHz cw radio-frequency quadrupole (RFQ) with associated high-power and lowlevel rf systems, a 52-magnet periodic lattice followed by a short high-energy beam transport (HEBT) and highpower $(670-\mathrm{kW} \mathrm{cw})$ beam stop. The rms beam emittance was measured prior to the installation of the 52-magnet lattice, based on wire-scanner measurements of the beam profile at a single location in the HEBT. New measurements with additional diagnostic hardware have been performed to determine the rms transverse beam properties of the beam at the output of the $6.7-\mathrm{MeV}$ LEDA RFQ.

The 52-magnet periodic lattice also includes ten beam position monitors (BPMs) evenly spaced in pairs of two. The BPMs provide a measure of the bunched beam current that exhibits nulls at different locations in the lattice. Model predictions of the locations of the nulls and the strength of the bunched beam current are made to determine what information this data can provide regarding the longitudinal beam emittance.
\end{abstract}

\section{INTRODUCTION}

The LEDA RFQ is described extensively elsewhere [1]. The primary objective of the 52-quadrupole-magnet FODO lattice is to provide a vehicle to measure phasespace halo in space charge dominated beams $[2,3]$. The quadrupole magnets are located every $21 \mathrm{~cm}$. Beam diagnostics, steering magnets and vacuum components are interspersed between the magnets. A schematic of the lattice showing the locations of the magnets and diagnostics is presented in Figure 1. The diagnostics are described in greater detail by Gilpatrick et al. [4].

The wire scanner located downstream from the fourth quadrupole magnet is used to measure transverse beam profiles for many different field settings of the first four quadrupole magnets. Measurements were made at beam currents of 16,50 and $75 \mathrm{~mA}$.

The bunched beam current has been measured at the ten BPMs for many different values of the beam current. Analyses of this data and comparison to the prediction of

*Work supported by U.S. DOE contract DE-AC04-96AL89607

†schulz@gat.com multi-particle simulation codes (LINAC) have been made. These comparisons indicate that the bunched beam current data provides information regarding the shape of the longitudinal beam distribution, a correlation between the longitudinal emittance and phase spread, and a measure of the rms energy spread of the debunched beam at the end of the beam line.

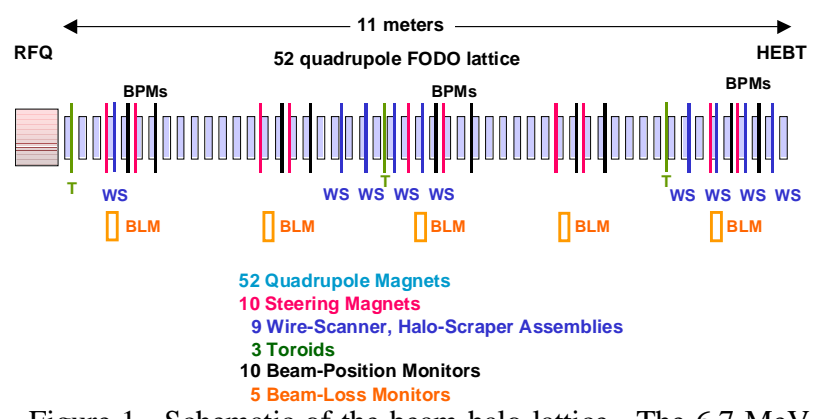

Figure 1. Schematic of the beam-halo lattice. The 6.7-MeV RFQ output $\mathrm{H}^{+}$beam enters from the left and exits to the right.

\section{TRANSVERSE EMITTANCE}

The procedure uses four quadrupoles installed at the output end of the RFQ, followed by a beam-profile diagnostic device that consists of both a thin movable carbon filament for measurement of the dense beam core, and scrapers for measurement of the lower-intensity halo. Transverse rms beam projections are measured as a function of the gradients of the four quadrupoles, and the data are used to determine the transverse Courant-Snyder parameters. The method involves using a fitting routine developed for both TRACE3D and LINAC that minimizes the $\chi^{2}$ of the difference between the measurements and the model predictions.

Each of the first four quadrupole magnets are set at field levels of $50 \%$ to $150 \%$ of their nominal values in steps of $25 \%$ while holding other quads at nominal design fields. This results in 17 data points for each transverse plane for each beam current. The measured beam distributions are analyzed off line to determine the full width at half of the maximum amplitude (FWHM) from which the rms width is determined.

In general the Courant-Snyder parameters determined using LINAC and TRACE3D are in good agreement with each other. The results are presented in Table 1 below. 
Table 1. Transverse Courant-Snyder Parameters as Predicted using TRACE3D and LINAC

\begin{tabular}{|l|c|c|c|c|c|c|}
\hline Current & \multicolumn{2}{|c|}{$16 \mathrm{~mA}$} & \multicolumn{2}{c|}{$50 \mathrm{~mA}$} & \multicolumn{2}{c|}{$75 \mathrm{~mA}$} \\
\hline Code & Linac & Trace & Linac & Trace & Linac & Trace \\
\hline$\varepsilon_{\mathrm{x}} \pi_{(\mathrm{mm}-\mathrm{mrad})}$ & 1.23 & 1.06 & 1.45 & 1.28 & 1.89 & 1.96 \\
\hline$\alpha_{\mathrm{x}}$ & 1.69 & 1.53 & 1.43 & 1.46 & 1.20 & 1.26 \\
\hline$\beta_{\mathrm{x}}(\mathrm{cm})$ & 20.4 & 19.5 & 20.1 & 25.5 & 19.4 & 24.3 \\
\hline$\varepsilon_{\mathrm{y}} \pi_{(\mathrm{mm}-\mathrm{mrad})}$ & 1.17 & 1.12 & 1.81 & 1.82 & 2.46 & 2.14 \\
\hline$\alpha_{\mathrm{y}}$ & -1.89 & -1.98 & -1.09 & -1.68 & -1.56 & -1.68 \\
\hline$\beta_{\mathrm{y}}(\mathrm{cm})$ & 55.7 & 59.1 & 35.7 & 52.2 & 48.2 & 55.5 \\
\hline
\end{tabular}

The beam emittances inferred from the experimental data are compared with predictions from PARMTEQM in Figure 2. The data points show very good agreement in the horizontal plane and suggest that the beam emittance in the vertical plane is larger than expected at higher beam currents. The larger vertical emittance is consistent with analyses presented at LINAC 2000 [5], although the beam emittances determined previously were analyzed on the basis of the rms width of the entire beam distribution. The analysis presented above, based on the FWHM of the beam distribution, does not take into account the observed "tails" in many of the beam distributions.

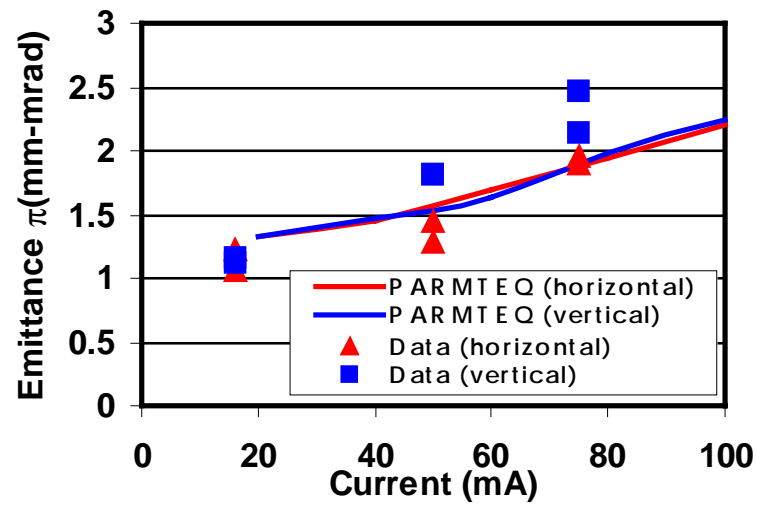

Figure 2: Comparison of the experimental transverse emittances with PARMTEQM predictions.

An extensive analysis of the shapes of the beam distribution is presented by Lysenko et al. [6] who reach the conclusion that either there is missing physics in the beam simulations or that the beam from the RFQ cannot be described by three Courant-Snyder parameters. Preliminary analyses indicate that beam distributions exhibiting nonlinear space charge filamentation can possibly describe the entire distribution, as well as the FWHM.

\section{BUNCHED BEAM CURRENT ANALYSIS}

The bunched beam current is a measure of the strength of the $350 \mathrm{MHz}$ signal in the beam pulse and can be determined from the phase spread of the beam distribution. The phase spread of the beam increases as the beam transports in the beam-halo lattice. In the absence of space charge forces, the increase in the phase spread can be determined directly from the energy spread of the beam and the shape of the longitudinal distribution. For high-current beams, space charge forces contribute significantly to increasing the phase and energy spread of the beam.

The bunched beam current was measured at ten locations in the beam-halo lattice. The data were obtained by averaging the measured bunched beam current over many pulses to reduce pulse-to-pulse jitter. Measurements were made at many values of the beam current ranging from 5 to $95 \mathrm{~mA}$. The data show that the location of the first null in the distribution is observed to appear further upstream as the beam current is increased. At the higher currents (> $50 \mathrm{~mA}$ ), a second null is observed in the 11 meter transport channel.

The amplitude of the bunched beam current can be expressed as a function of the phase spread of the beam as presented in Equation 1 for a parabolic longitudinal phase distribution [7].

$$
A=3\left(\left(\frac{\sin (a)}{a^{3}}\right)-\left(\frac{\cos (a)}{a^{2}}\right)\right)
$$

Here, $a=\pi \mathrm{W} / \mathrm{T}$, and $\mathrm{W}$ is the full phase width at the base of the bunch and $T$ is the bunch spacing $(T=1 / f)$.

The rms phase spread of the beam is calculated at many locations along the beam-halo lattice using LINAC. A 6$\mathrm{D}$ waterbag distribution has been assumed for the initial distribution at the end of the RFQ. This distribution gives the same result as the beam distributions from PARMTEQM simulations of the RFQ. The full phase spread at the base of a parabolic distribution is 4.472 times the rms phase spread. The beam phase distributions from LINAC are consistent with parabolic distributions, although due to the finite number of particles, the full width at the base of the phase distribution is typically 4.25 rms. This value is used in the calculations of the bunched beam current. Although the analysis is consistent with a parabolic distribution, other phase distributions cannot be ruled out without further study.

LINAC is used to determine the values of $\varepsilon_{\mathrm{z}}$ and $\beta_{\mathrm{z}}$ that provide the best fit to the bunched beam current data. Figure 3 shows a typical fit to the data at $95 \mathrm{~mA}$.

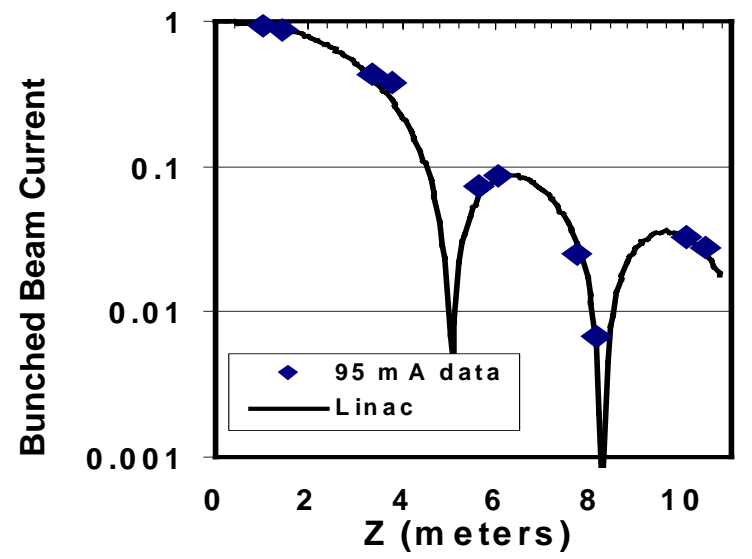

Figure 3: Normalized bunched beam current at $95 \mathrm{~mA}$ compared to typical LINAC fit. 
The longitudinal beam-phase ellipse from the RFQ, as predicted by PARMTEQM, is essentially an upright ellipse and no attempt has been made to determine $\alpha_{z}$ from the data. The analysis does not provide a unique value for $\varepsilon_{z}$ and $\beta_{z}$, but rather defines a correlation between $\varepsilon_{z}$ and $\beta_{z}$ that provide equally good fits to the data. The results of analyses performed at 78 and $95 \mathrm{~mA}$ are presented in Figures 4 and 5 respectively. These figures show that the correlation between the values of $\varepsilon_{\mathrm{z}}$ and $\beta_{z}$ is approximately linear with a negative slope. The lines in the figures connecting the points are to guide the eye. The data at $95 \mathrm{~mA}$ support a much greater range of values than at $78 \mathrm{~mA}$. This is because a smaller initial phase spread coupled with a larger emittance (larger energy spread) produces the same result as a large initial phase spread and smaller emittance due to the effects of space charge forces. The effects of space charge increase the range of the correlation. Analysis of the data at $10 \mathrm{~mA}$ indicates that a positive slope in the correlation between $\varepsilon_{z}$ and $\beta_{\mathrm{z}}$ provides the best fit to the data.

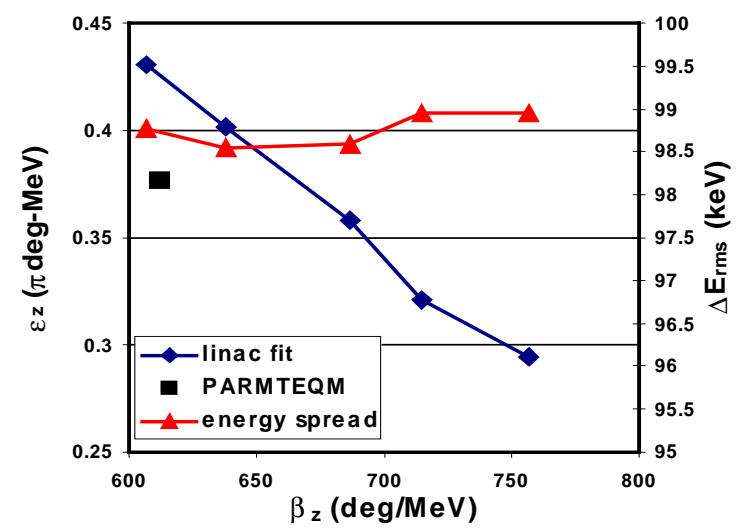

Figure 4. Longitudinal emittance and energy spread at $78 \mathrm{~mA}$.

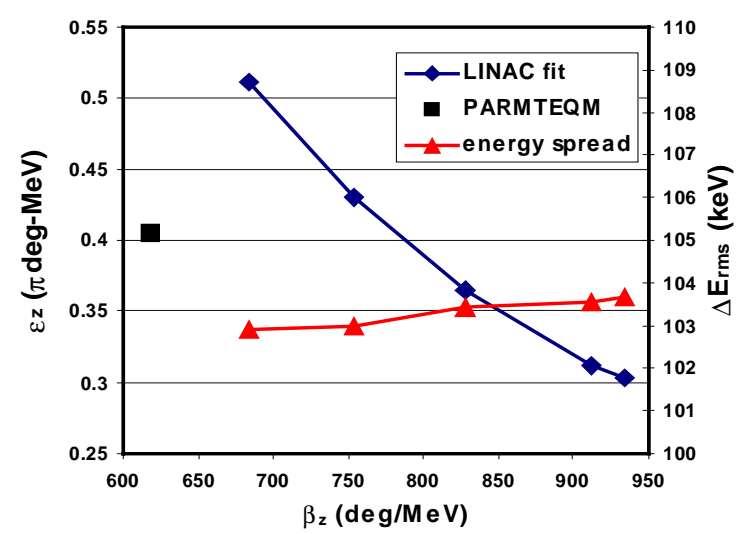

Figure 5. Longitudinal emittance and energy spread at $95 \mathrm{~mA}$

The PARMTEQM predictions for $\varepsilon_{\mathrm{z}}$ and $\beta_{\mathrm{z}}$ and the predicted rms energy spread at the end of the 11-meter lattice are also plotted in Figures 4 and 5. The results are in better agreement with PARMTEQM simulations at 78 $\mathrm{mA}$ than at $95 \mathrm{~mA}$. The analyses presented in Figures 4 and 5 show that the energy spread at the end of the beamhalo lattice is very well defined by the bunched beam current measurements. The final energy spreads at 78 and $95 \mathrm{~mA}$ are about 98.7 and $103 \mathrm{keV}$, respectively. The uncertainty in these values is very small as the beam is fully debunched at the end of the channel and the energy spread is no longer increasing due to space-charge forces. The rms energy spread in the beam-halo lattice as predicted by LINAC is plotted in Figure 6 for initial values of $\varepsilon_{\mathrm{z}}$ and $\beta_{\mathrm{z}}$ that provide a good fit to the bunched beam-current measurements. Results are presented for 10,78 and $95 \mathrm{~mA}$.

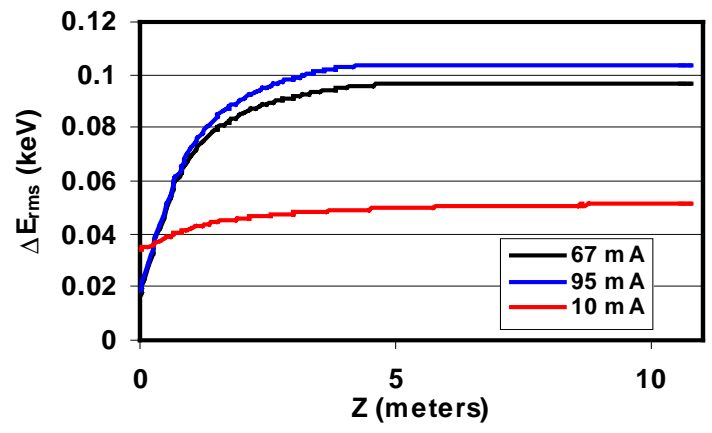

Figure 6. RMS energy spread along the beam-halo lattice as predicted by LINAC

\section{SUMMARY}

The rms transverse beam properties at the exit of the RFQ inferred from quadrupole scan beam profile measurements are in relatively good agreement with PARMTEQM simulation of the RFQ. The overall shapes of the distributions are not well understood at the present time. Bunched beam-current measurements of the RFQ beam are able to provide significant information regarding the longitudinal beam properties.

\section{REFERENCES}

[1] L.M. Young., "Operations of the LEDA ResonantlyCoupled RFQ," this conference.

[2] T. Wangler, et al., "Experimental Study of ProtonBeam Halo Induced by Beam Mismatch in LEDA," this conference.

[3] P. L. Colestock, et al., "Measurements of Halo Generation for a Proton Beam in a FODO Channel," this conference.

[4] J. D. Gilpatrick, et al., "Experience with the LowEnergy Demonstration Accelerator (LEDA) Halo Experiment Beam Instrumentation," this conference.

[5] M. E. Schulze, et al., "Beam Emittance Measurements for the Low-Energy Demonstration Accelerator RadioFrequency Quadrupole," Proc. LINAC2000 (Monterey, 21-25 August 2000), pp. 575-577.

[6] W. P. Lysenko, et al., "Characterizing Proton Beam of 6.7 MeV LEDA RFQ by Fitting Wire-Scanner Profiles to 3-D Nonlinear Distributions," this conference.

[7]R. E. Shafer, "Beam Position Monitoring", 1989 Accelerator Instrumentation Workshop (BNL), AIP Conference Proceeding 212 (1990). See Table 4.1. 